\title{
Battery impedance spectroscopy using bidirectional grid connected converter
}

\author{
SHIMUL KUMAR DAM* and VINOD JOHN \\ Department of Electrical Engineering, Indian Institute of Science, Bangalore 560012, India \\ e-mail: shimul@ee.iisc.ernet.in; vjohn@ee.iisc.ernet.in
}

MS received 15 March 2016; revised 17 August 2016; accepted 28 September 2016

\begin{abstract}
Battery impedance can provide valuable insight into the condition of the battery. Commercially available impedance measurement instruments are expensive. Hence their direct use in a battery management system is not justifiable. In this work, a $3-\mathrm{kW}$ bi-directional converter for charging and discharging a battery bank has been implemented with the capability of impedance measurement. The converter is grid connected and controlled to operate at unity power factor. Additional requirements on filter design and control structure of battery converter for impedance measurement are discussed. An algorithm has been developed to measure impedance by frequency sweep, avoiding transients. The measured impedance has been compared to that from a commercially available impedance measurement equipment and is shown to have a good match.
\end{abstract}

Keywords. Impedance spectroscopy; grid connection; battery converter; state of charge; health monitoring.

\section{Introduction}

Batteries play an important role as energy storage devices for renewable energy sources, electric vehicle and many other applications. A battery bank is interfaced to load through a power converter, which controls the charging and discharging of the battery. Often the battery bank is responsible for most of the cost of the storage system. Battery should be utilized properly so that maximum battery life can be obtained. Hence, battery health should be monitored continuously. For monitoring battery health in battery management system one needs to track two variables - state of charge (SOC) and state of health ( $\mathrm{SOH}$ ). SOC is defined as the ratio of available charge at a point of time to total charge capacity of the battery. SOH is defined as the ratio of present charge storage capacity to initial charge storage capacity when the battery was new [1].

The method of battery health monitoring is preferred to be non-intrusive. Such a method needs a relation between SOC and SOH with easily obtainable parameters like terminal voltage, current and temperature. Fundamental methods of energy conversion in battery have been modelled in electro-chemical domain. Although this model gives good estimate, it requires high computation resources,

The authors would like to acknowledge the financial support from Department of Heavy Industry (DHI), Government of India, under the project Offline and realtime simulator for Electric Vehicle/Hybrid Electric Vehicle systems.

*For correspondence long time and battery specific information [2]. hence it is not suitable for health monitoring. On the other hand, datadriven approaches like Neural Network, Support Vector Machine and Fuzzy Logic do not need any model and give reasonable accuracy [3]. But they need a large amount of training data and can lead to incorrect result if the data-set is biased [3, 4]. Equivalent circuit models make use of simplified electrical circuit to model battery response. These models are simple but cannot predict battery response under varying load [2,3]. To address these issues, other approaches like Kalman-filter and impedance-spectroscopy-based-estimation have been developed, which use simplified model as well as run-time data to take care of aging effect and uncertainty in modelling [3]. It is stated in $[4,5]$ that Kalman-filter-based estimation processes may diverge under certain conditions and their accuracy is dependent on initial values. Impedance-spectroscopy-based estimation uses simple-small signal linear electrical model of the battery. Hence it is easier to implement in hardware. However, it suffers from temperature dependence [6], long measurement time and costly measuring equipment [4]. Despite these drawbacks, it has been used for estimating SOC and SOH in literature [6-9].

In this work, the necessary hardware and algorithm have been developed so that impedance spectroscopy can be performed by a bidirectional battery charger, shown in figure 1 without the need for a dedicated instrument for it. A similar work has been reported in [10]. This work describes the necessary control structure for controlling low-amplitude perturbation and an algorithm suitable for fast impedance measurement in low-frequency range, 

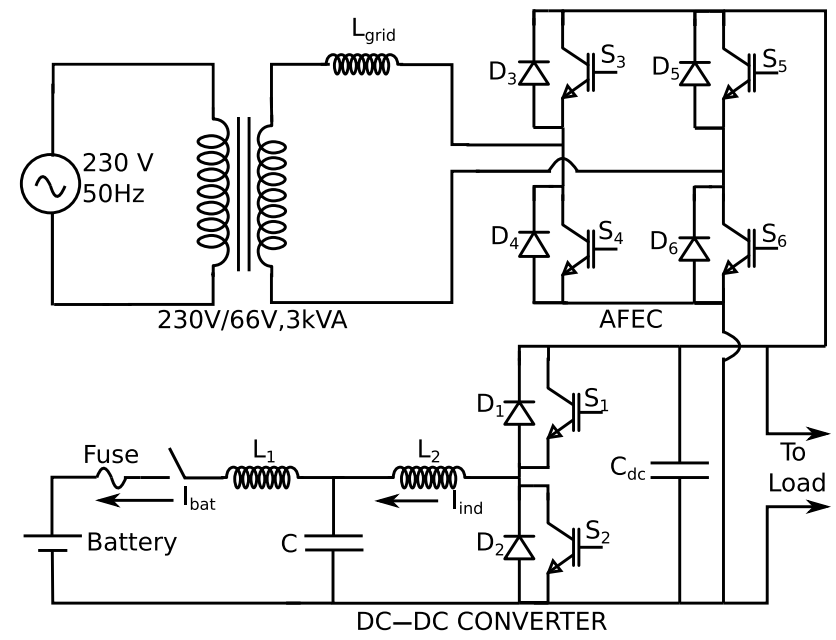

Figure 1. Converter topology for interaction between grid and battery.

which were not reported in those publications. A simple measurement circuit also has been used to measure battery impedance in a desired frequency range. The necessity of low-amplitude perturbation at low frequency has been discussed in detail. Another major difference of topology of this work from previously mentioned works is that this topology employs a grid-connected active front end converter (AFEC) to avoid wastage of energy during testing of battery. The organization of the paper is as follows. Section 2 explains the hardware topology of the grid-connected converter. Important aspects of implementing impedance spectroscopy and small signal model have been discussed in section 3. Section 4 explains the control structures. Section 5 describes necessary measurement circuit and algorithm and section 6 contains experimental results.

\section{Converter topology}

The converter topology chosen is a versatile topology, shown in figure 1. It can be used either as grid-connected battery testing and characterization set-up or as a backup system of grid-connected load or storage system for renewable energy sources. For testing and characterization applications this topology allows sending back power to grid while battery is discharging, thus saving considerable energy. For backup and storage application, the load or the renewable source is connected to the dc bus through another dc-dc converter. This part is not shown in figure 1 . The battery connected in this system can supply the load in case the grid fails or it can store the excess energy produced by renewable source to feed the grid later when power demand is high, thus improving the power quality. The topology has two back-to-back connected converters: an AFEC and a dc-dc converter.

\subsection{AFEC}

This converter has H-bridge topology connected to grid through an inductor and a step-down transformer. The inductor is designed to minimize harmonics injected to the grid. This converter plays the role of interfacing the grid with dc bus. It takes power from grid at unity power factor when the battery is being charged, and feeds power to grid when battery discharges. For this purpose the controllers for AFEC must know the grid phase. The grid phase is estimated by a phase-locked loop [11]. The dc bus voltage is chosen based on battery voltage rating. A step-down transformer is used to bring down the grid voltage so that dc bus voltage is sufficient for inverter operation.

\section{$2.2 D c-d c$ converter}

The dc-dc converter controls the charging and discharging of the battery. Hence this converter plays an important role for testing and characterization of the battery. Any desired load profile can be generated by this converter. However, the load should be within the safe range of operation specified by the manufacturer to avoid rapid deterioration of battery health.

Battery manufacturers specify that current in the battery should be dc; ac components in current can reduce battery life. Hence the filter at the output of the dc-dc converter should be designed to keep current ripple to a minimum value. An LC filter would be sufficient for a resistive or inductive load. But battery has large double layer capacitance. Hence the ripple current is shared by filter capacitor and battery. Thus, an LCL filter is necessary for proper attenuation of switching frequency component. Appropriate damping must be provided to damp out resonant frequency oscillations. A split capacitor damping scheme is adopted here as shown in figure 2 [12].

The objective of LCL filter design is to minimize current ripple in battery such that battery health is not affected by high-frequency current. However, LCL filter in a converter used for impedance measurement, must also ensure that current ripple magnitude is less compared with current perturbation during impedance spectroscopy.

The designing of the power circuit filter to have low ripple current is necessary for measurement of impedance. Switching frequency is not a multiple of the frequency of measurement. Hence, switching frequency ripple cannot be eliminated by Fourier series analysis.

The switching frequency ripple present in measured voltage and current must be filtered out by a low-pass filter in measurement circuit. The cut-off frequency of the lowpass filter has been set to $1 \mathrm{kHz}$ (one decade higher than the highest measurement frequency of $100 \mathrm{~Hz}$ ). Hence, this cut-off frequency is 1.3 decade below the switching frequency $(20 \mathrm{kHz})$. A second-order low-pass filter can provide attenuation of more than $50 \mathrm{~dB}$. The magnitude of 


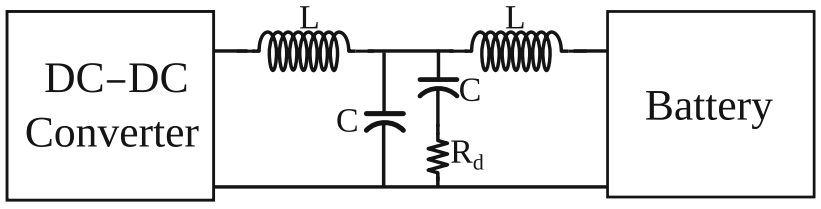

Figure 2. Split capacitor damping scheme for LCL filter.

switching ripple in measured battery current and voltage must be low, so that it becomes negligible after $50 \mathrm{~dB}$ attenuation.

In this work, current perturbation amplitude is $100 \mathrm{~mA}$ and switching ripple in battery current is chosen to be 5 $\mathrm{mA}$ peak-peak. Thus, after attenuation of $50 \mathrm{~dB}$ in measurement circuit, the measured switching ripple becomes less than $0.02 \%$ of amplitude of measured current perturbation.

Hence, impedance measurement imposes tight current ripple requirement on the power circuit filter design. Thus, the filter size increases and filter becomes bulky in case of an L filter. Use of LCL filter, instead of L filter, reduces filter size and cost. Although system cost is increased, it may be justified in terms of benefits of impedance-based health monitoring.

In general, low battery ripple current is desirable from a high-performance charger, even though there are no specific standards to be met. Hence the power circuit can be considered as a high-performance charger.

\section{Impedance spectroscopy}

Battery terminal voltage and current exhibit non-linear internal characteristics. However, battery can be assumed to be linear for small-signal perturbation about an operating point. Under this condition, it can be assumed to be an ideal voltage source with an impedance in series. This impedance is measured in impedance spectroscopy over a frequency range and fitted to an impedance model to extract model parameters, which are functions of SOC and $\mathrm{SOH}$ of the battery [13]. Impedance measuring equipments are commercially available. But owing to their very high cost, their usage is limited to research and development. Solartron CellTest System [14] is one such instrument, which is used in this work to compare the obtained results. One of the aims of this work is to perform impedance spectroscopy with a VSI-based two-level power converter instead of using any dedicated measurement equipment.

\subsection{Small-signal perturbation}

In order to measure the impedance at a certain frequency, a sinusoidal perturbation of current has to be produce, causing a voltage perturbation at that frequency. Then the magnitude and phase of impedance can be measured. However, the perturbation should be small enough so that the voltage perturbation amplitude is less than $10 \mathrm{mV} / \mathrm{cell}$ $[1,6]$ where the nominal cell voltage of lead-acid battery is $2 \mathrm{~V}$. This limit ensures that the measurement is carried out under linear condition.

Impedance can be measured by producing voltage perturbation, causing current perturbation, or producing a current perturbation, causing a voltage perturbation. In either way the power converter must apply sinusoidal voltage of amplitude less than $10 \mathrm{mV} / \mathrm{cell}$ in addition to a dc voltage. Hence output voltage resolution of the converter must match the requirement to produce such a small perturbation.

\subsection{Frequency range}

The frequency range of measurement is an important criterion. During the entire time of measurement, drift in system should be negligible. Hence the measurement should be as fast as possible. Hence, higher frequency range is preferable in terms of measurement time. In [9], the transition frequency at which impedance is purely resistive is used to estimate SOC. This frequency lies in the range of a few hundred $\mathrm{Hz}$ and it is suitable for quick measurement. However, [15] suggests that the lower frequency of impedance measurement is suitable for characterization and the lowest frequency should be less than $10 \mathrm{mHz}$ for studying the aging effect. To find out usefulness of measurements at different frequencies, the impedance of a $12 \mathrm{~V}, 9$ Ah VRLA battery is measured by a standard commercial equipment Solartron CellTest System [14] in the frequency range $1 \mathrm{mHz}-1 \mathrm{kHz}$ at different SOCs. The results are plotted in figure 3 .

It can be observed that variation of impedance with respect to SOC is more over frequency range below $100 \mathrm{mHz}$. This means that lower frequency range contains more information about battery condition. However, measurement over lower frequency range requires large amount of time and risks possible system drift. Thus, selection of the lowest frequency of the frequency range is a trade-off between measurement time and accuracy.

Although higher frequency range is attractive in terms of speed of measurement, impedance is inductive over that range. Inductance of battery is attributed to electrodes and connecting leads, which do not change with battery condition. Hence, measurement at higher frequency does not provide battery internal information.

The highest frequency of measurement is chosen to be $100 \mathrm{~Hz}$ (experimental results show only upto $50 \mathrm{~Hz}$ ). Switching frequency of the converter is chosen based on filter requirement and switching loss. This switching frequency $(20 \mathrm{kHz})$ is capable of injecting $100 \mathrm{~Hz}$ sinusoidal current in the battery. 

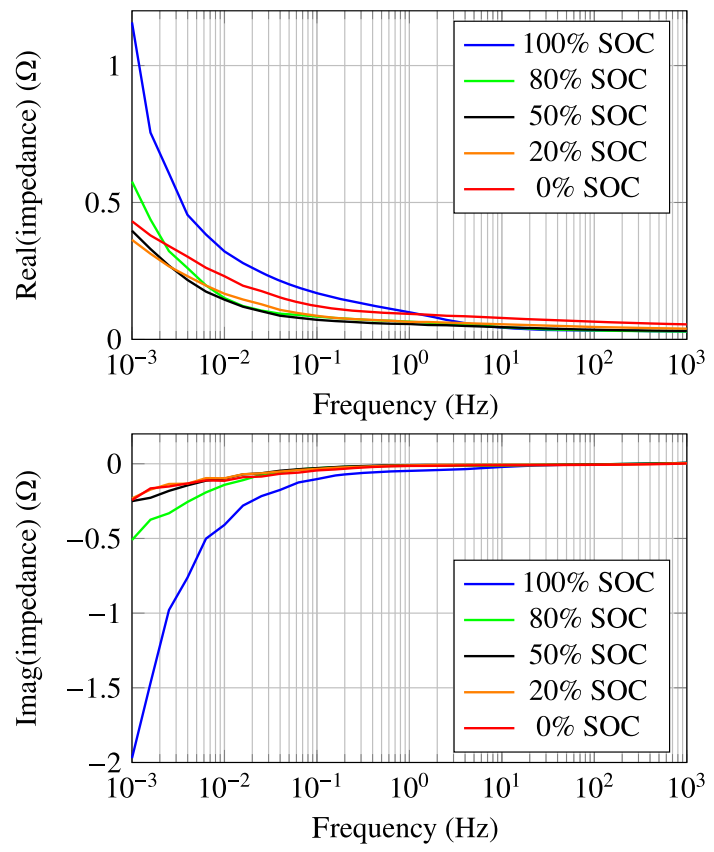

Figure 3. Variation of real and imaginary part of impedance with frequency at different SOC.

\subsection{Small-signal model}

The measured impedance data are fitted to a linear model of cell impedance. This model can be empirical or derived from physical processes in the battery. However, physicalprocess-based model provides better fitting to experimental data [13]. A widely accepted model of battery cell is the finite Warburg impedance model [6], consisting of RC networks and a frequency-dependent impedance. The model is shown in figure 4.

Here, the impedance ' $Z_{w}$ ' is the finite Warburg impedance. The expression for this impedance that reflects the diffusion through a layer of electrolyte is given in (1):

$$
Z_{w}=\frac{W_{b}(1-j) \operatorname{coth}\left(\mathrm{W}_{\mathrm{c}}(1+\mathrm{j}) \sqrt{\omega}\right)}{\sqrt{\omega}} .
$$

The suitability of this model is shown for a $12 \mathrm{~V}, 9 \mathrm{Ah}$ VRLA battery in figure 5. Impedance data at $70 \%$ SOC are fitted to the finite Warburg model. Figure 5 shows that the fit is good with rms error $4.88 \%$ for real part and $5.05 \%$ for imaginary parts. If the finite Warburg impedance is

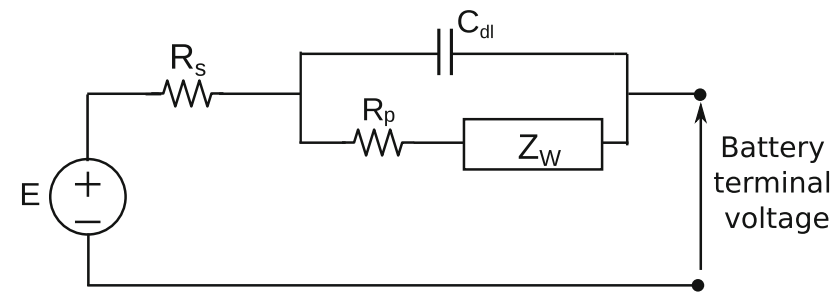

Figure 4. Small signal model of the battery with finite Warburg impedance network [1]. replaced by a capacitor then the best case rms errors for curve fitting become $18.66 \%$ and $22.35 \%$ for real and imaginary parts, respectively.

\section{Converter control structure}

The controller of AFEC has the task of taking power from or feeding power to grid at unity power factor. The controller of the $\mathrm{dc}-\mathrm{dc}$ converter controls the charging and discharging of the battery and facilitates impedance spectroscopy.

\subsection{Controller for AFEC}

The control structure of AFEC is shown in figure 6. The objectives of AFEC are to regulate dc bus voltage in a tight range and to control sinusoidal input current with low THD and at unity power factor. The inner loop controls the current by a proportional resonant controller. The outer loop controls the dc bus voltage using a PI controller [16].

In figure $6, V_{g(f f)}$ is a feed-forward term derived from measured grid voltage to reduce controller output swing, $I_{f d b}$ is measured grid current, $V_{d c(f d b)}$ is the measured dc bus voltage and $\theta_{p l l}$ is the phase of grid voltage estimated by Synchronous Reference Frame PLL. This PLL is preceded by a Second-Order Generalized Integrator (SOGI) block to generate orthogonal vector and to filter out unwanted frequencies. The bandwidth of PLL has been chosen to be 50 $\mathrm{Hz}$ as suggested in [11]. The PR controller is implemented by the structure shown in figure 7 where $T_{s m p}$ is the sampling interval.

' $\omega_{o}$ ' is the grid frequency estimated by the PLL. The selection of parameters of PR controller is preceded by designing a PI controller of desired bandwidth; later PI parameters are converted to PR parameters as described by [17].

\subsection{Controller for $d c-d c$ converter}

The controller of the dc-dc converter controls the battery voltage only at the last part of charging and it controls battery current during remaining charging-discharging cycle. Impedance spectroscopy is also performed in current control mode. During the current control mode, the reference given to the controller is shown in figure 8. For charging and discharging the ac reference is turned off. During impedance spectroscopy, ac reference that is turned on and dc reference can be either zero for impedance measurement under no load or a desired value for impedance measurement under load.

As discussed earlier, impedance measured over lower range of frequencies has higher importance for monitoring battery health. Hence, the frequency range of ac reference 


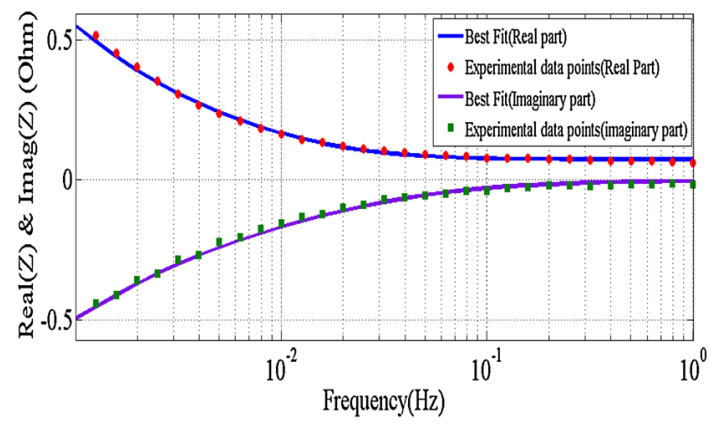

Figure 5. Measured impedance data points and curve fitting at $70 \%$ SOC of a $12 \mathrm{~V}, 9$ Ah VRLA battrey.

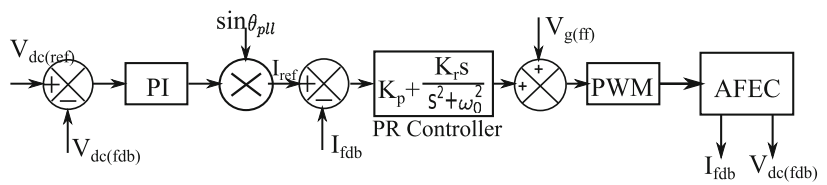

Figure 6. Double loop control structure of grid connected bidirectional active front end converter.

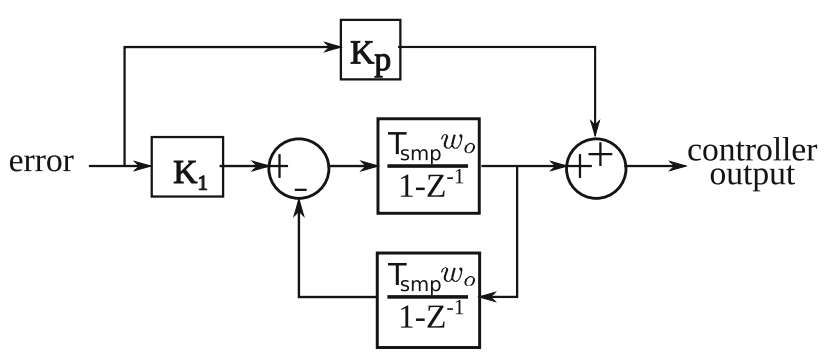

Figure 7. Implementation of PR controller in discrete domain.

typically lies between a few $\mathrm{mHz}$ and a few hundreds of $\mathrm{Hz}$. The controller should ensure zero or negligible steadystate error at $\mathrm{dc}$ and in this frequency range.

Although frequency range of reference is low, the controller should be as fast as possible without any overshoot. Faster response of controller causes the transients to die down quickly, thus saving valuable time, especially at low frequency.

LCL filter of the dc-dc converter is designed to have large attenuation at switching frequency since high-frequency ripple is detrimental to battery health [18]. The resonant frequency of such filter is on the order of a few hundred $\mathrm{Hz}$. If a single loop control system is designed to control the battery current by taking it as a feedback, as shown in figure 8a, the open loop transfer function has $180^{\circ}$ phase lag around resonant frequency of the LCL filter and control bandwidth will be limited to that frequency [19]. For example, the LCL filter with component values as shown in table 1 has a resonant frequency of $288 \mathrm{~Hz}$. Hence, the maximum bandwidth can be only $288 \mathrm{~Hz}$.
On the other hand, if the converter side inductor current is controlled as shown in figure $8 \mathrm{~b}$, then higher bandwidth can be achieved [19]. In this work, this control loop is controlled by a PI controller to have a bandwidth of $1.9 \mathrm{kHz}$ as depicted in the Bode plot of the open-loop transfer function between $I_{\text {ind }}$ and $I_{\text {ind }}^{*}$ as shown in figure 9. Now, the transfer function $I_{b a t}(s) / I_{\text {ind }}(s)$ has unity gain for lower range of frequency. Hence, this scheme may be sufficient for $\mathrm{dc}$ reference tracking in terms of steady-state error, but it leads to reduction in bandwidth. This reduction can be seen clearly from the Bode plot shown in figure 10 where the blue dotted lines are magnitude and phase plots of closed-loop transfer function between $I_{\text {ind }}$ and $I_{\text {ind }}^{*}$ and the green continuous lines are the magnitude and phase plots between $I_{b a t}$ and $I_{\text {ind }}^{*}$. In the magnitude plot, bandwidth is given by the frequency up to which the gain is more than $-3 \mathrm{~dB}$. Hence, it can be noticed that even if the $I_{\text {ind }}$ control loop has bandwidth of $1.9 \mathrm{kHz}$, the actual bandwidth for controlling the battery current $I_{b a t}$ is $320 \mathrm{~Hz}$, thus, the improvement in bandwidth is lost. Moreover, in the range of $80-320 \mathrm{~Hz}$. the gain is more than unity. This means that in this range of frequency, battery current will be more than the reference current, which may exceed the upper limit of ac excitation for ensuring validity of linear battery system assumption. All these limitations lead to the use of a double-loop control structure.

The double-loop control structure is shown in figure 8c. With this structure and using the PI controller and lead compensator, a bandwidth of $800 \mathrm{~Hz}$ is achieved for control of $I_{b a t}$. The magnitude and phase of the open loop transfer function between $I_{b a t}$ and $I_{b a t}^{*}$ are is shown by the blue dotted line in figure 11. This figure also shows that a phase margin of $70^{\circ}$ is achieved. Now, it can be observed from this magnitude plot (blue dotted line) that the gain is less than $40 \mathrm{~dB}$ in the frequency range $10-800 \mathrm{~Hz}$. At least $40 \mathrm{~dB}$ gain is required at a certain frequency if steady-state error should be lower than $1 \%$ at that frequency.

When impedance spectroscopy is performed by the single sine excitation method, the ac current reference contains only one frequency. At that frequency the steady-state error should be very low. Hence, high gain at a particular frequency can be achieved by using a proportional resonant controller. This controller is connected in series with the existing outer loop PI controller. This combination of PI and PR controllers is termed as the Proportional Integral Resonant controllers or PIR controller. Now, the inclusion of PR controller should not change the bandwidth and phase margin of the system. Hence the proportional gain of the PR controller is taken as unity. The transfer function of the PR controller is

$$
G_{P R}=1+\frac{K_{r} s}{s^{2}+\omega_{0}^{2}} .
$$

This transfer function can be expressed in the following form from the point of view of implementation shown in figure 7 , 


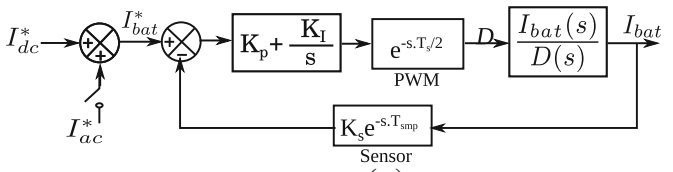

(a)

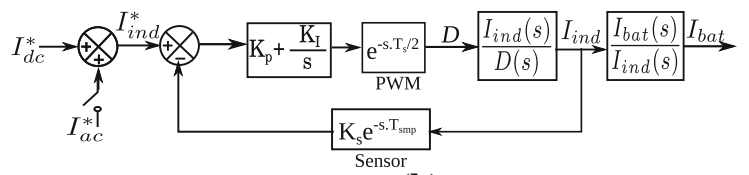

(b)

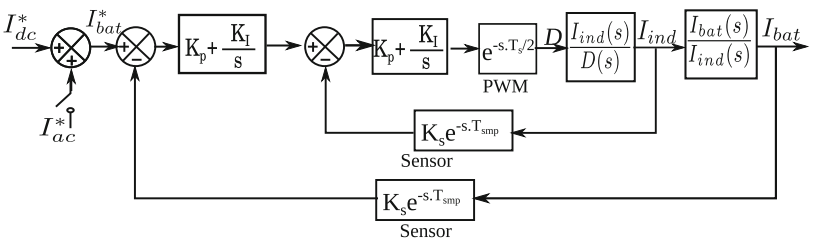

(c)

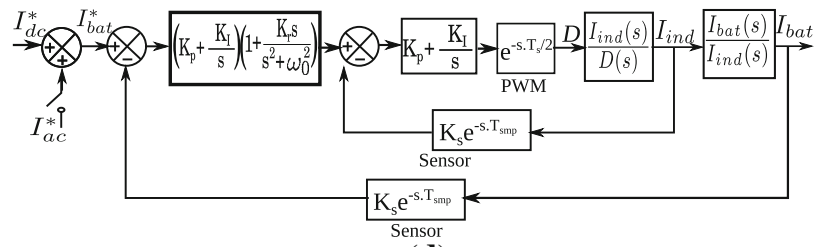

(d)

Figure 8. Control structures of dc-dc converter, (a) single loop ' $I_{\text {bat }}$ ' control, (b) single loop ' $I_{\text {ind }}$ ' control, (c) double loop control with PI controller and (d) double loop control with PIR controller in outer loop.

Table 1. Bidirectional dc-dc converter parameters.

\begin{tabular}{lcc}
\hline Quantity & Symbol & Value \\
\hline dc bus voltage & $V_{d c}$ & $120 \mathrm{~V}$ \\
Filter inductance & $L$ & $650 \mu \mathrm{H}$ \\
Filter capacitance & $C$ & $470 \mu \mathrm{F}$ \\
Damping resistance & $r_{d}$ & $1 \Omega$
\end{tabular}

$$
\begin{gathered}
G_{P R}=1+\frac{K_{1} \omega_{0} s}{s^{2}+\omega_{0}^{2}}, \\
K_{1}=\frac{K_{r}}{\omega_{0}} .
\end{gathered}
$$

The PR controller is implemented by forward and backward Euler approximation for simplicity in implementation. This implementation suffers from the limitation of shift in resonant frequency ' $\omega_{0}$ ' due to discretization and quantization [20]. Fortunately, the PR controller is capable of providing high gain in the vicinity of resonant frequency. Hence, the parameter ' $K_{1}$ ' in (3) must be chosen such that PR controller can provide sufficient gain at resonant frequency even if it shifts from its ideal value.
In order to design the PR controller the maximum possible shift in resonant frequency need to be determined first. The shifted resonant frequency due to discretization [20] is given in Eq. (5), where ' $T_{s}$ ' is sampling time;

$$
\begin{gathered}
\omega_{0}^{\prime}=\frac{2}{T_{s}} \sin ^{-1}\left(\frac{\omega_{0} T_{s}}{2}\right) \\
\Rightarrow \frac{\omega_{0}^{\prime}}{\omega_{0}}=\frac{\sin ^{-1}\left(\frac{\omega_{0} T_{s}}{2}\right)}{\left(\frac{\omega_{0} T_{s}}{2}\right)} .
\end{gathered}
$$

The ratio of shifted to ideal ' $\omega_{0}$ ' in Eq. (6) becomes 1 when ' $\omega_{0} T_{s}$ ' is small. Hence, deviation of resonant frequency is maximum for the maximum value of ' $\omega_{0}$ '. For the worst case, the ratio of maximum shifted ' $\omega_{0}$ ' to ideal ' $\omega$ ' is given by

$$
\left.\frac{\omega_{0}^{\prime}}{\omega_{0}}\right|_{\omega_{0}=\omega_{0(\max )}}=\frac{\sin ^{-1}\left(\frac{\omega_{0(\max )} \mathrm{T}_{\mathrm{s}}}{2}\right)}{\left(\frac{\omega_{0(\max )} T_{s}}{2}\right)} .
$$

Hence, the maximum relative frequency deviation due to discretization is given by

$$
K_{d i s}=\left|\frac{\omega_{0}^{\prime}-\omega_{0}}{\omega_{0}}\right|_{\omega_{0}=\omega_{0(\max )}}=\left|\frac{\sin ^{-1}\left(\frac{\omega_{0(\max } \mathrm{T}_{\mathrm{s}}}{2}\right)}{\left(\frac{\omega_{0(\max )} T_{s}}{2}\right)}-1\right| .
$$

There is further shift in resonance frequency due to quantization. This shift of resonance frequency is caused mainly by truncation of the product ' $\omega_{0} T_{s}$ ' due to a finite number of bits of variable in which it is stored [20]. The variable used here is a 16-bit variable in which 14 bits are used for storing fraction, which means the per unit is represented by $2^{14}$. The maximum truncation error can be 1 LSB, i.e., $1 / 2^{14}$. Hence, the maximum relative shift in resonant frequency ' $\omega_{0}$ ' is given by

$$
\left|\frac{\omega_{0}^{\prime}-\omega_{0}}{\omega_{0}}\right|=\frac{\frac{1}{2^{14}}}{\omega_{0} T_{s}} .
$$

This error is maximum when frequency is the lowest. From figure 11, it can be observed that the PI controller alone can provide $40 \mathrm{~dB}$ gain for frequency below $10 \mathrm{~Hz}$. Hence the lowest frequency up to which the PR controller is supposed to provide gain is taken as $10 \mathrm{~Hz}$. For this minimum ' $\omega_{0}$ ', i.e., ' $\omega_{0(\text { min })}$, the maximum relative frequency deviation is given by

$$
K_{q n t}=\left|\frac{\omega_{0}^{\prime}-\omega_{0}}{\omega_{0}}\right|_{\omega_{0}=\omega_{0(\text { min })}}=\frac{\frac{1}{2^{14}}}{\omega_{0(\text { min })} T_{s}} .
$$

Hence, combining both frequency shifts due to discretization and quantization, the upper limit of relative shift in frequency is given by 

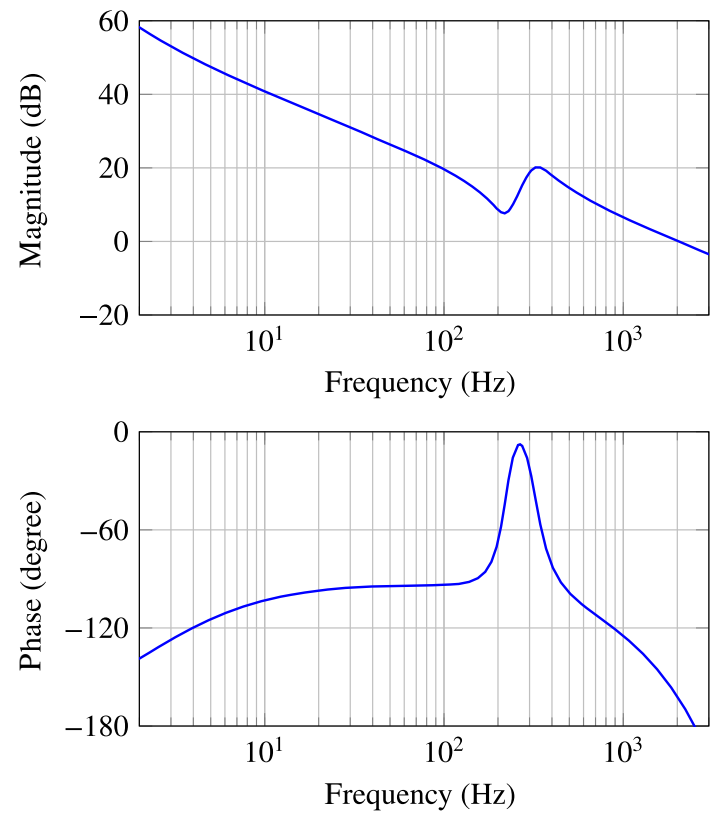

Figure 9. Magnitude and phase plots of open loop transfer function between ' $I_{\text {ind }}$ ' and ' $I_{\text {ind }}^{*}$ '.
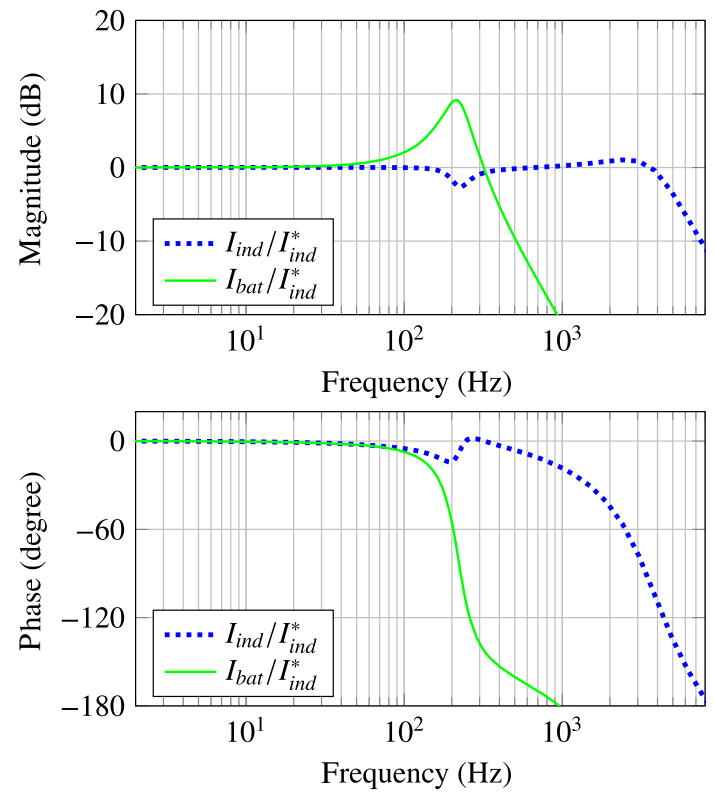

Figure 10. Magnitude and phase plots of inner loop transfer function $I_{\text {ind }}(s) / I_{\text {ind }}^{*}(s)$ under close loop condition (blue dotted line) and transfer function $I_{b a t} / I_{i n d}^{*}$ with open outer battery current loop and closed inner current loop (continuous green lines).

$$
K_{\text {shift }}=K_{d i s}+K_{q n t} .
$$

Let ' $\omega_{m}$ ' be a frequency near resonant frequency ' $\omega_{0}$ ' such that

$$
\left|\frac{\omega_{m}-\omega_{0}}{\omega_{0}}\right|=K_{\text {shift }}
$$

Hence, at $\omega=\omega_{m}$, the PR controller should provide sufficient gain ' $m$ '. Thus, at frequency ' $\omega_{m}$ ', the gain provided by PR controller is given by

$$
\begin{gathered}
G_{P R}\left(j \omega_{m}\right) \mid=M \\
\Rightarrow 1+\frac{\left(K_{1} \omega_{0} \omega_{m}\right)^{2}}{\left(\omega_{0}^{2}-\omega_{m}^{2}\right)^{2}}=M^{2} \\
K_{1}=\sqrt{M^{2}-1}\left|\frac{\omega_{0}}{\omega_{m}}-\frac{\omega_{m}}{\omega_{0}}\right| .
\end{gathered}
$$

The ratio $\omega_{0} / \omega_{m}$ can be found out from Eq. (12) and using that value, ' $K_{1}$ ' can be found out from Eq. (14).

Another value of ' $K_{1}$ ' can be obtained by phase consideration. The PR controller also provides phase lag at gain cross-over frequency, ' $\omega_{g}{ }^{\prime}$, reducing phase margin. Let the permissible reduction in phase margin due to incorporation of PR controller be ' $\theta$ '; then

$$
\begin{gathered}
\angle G_{P R}\left(j \omega_{g}\right)=-\theta \\
\Rightarrow-\tan \theta=\frac{K_{1} \omega_{0} \omega_{g}}{\omega_{0}^{2}-\omega_{g}^{2}} \\
\Rightarrow K_{1}=\tan \theta\left[\frac{\omega_{g}^{2}-\omega_{0}^{2}}{\omega_{0} \omega_{g}}\right] \\
\Rightarrow K_{1}=\tan \theta\left[\frac{\omega_{g}}{\omega_{0}}-\frac{\omega_{0}}{\omega_{g}}\right] .
\end{gathered}
$$

From Eq. (17), the phase lag provided by PR controller at ' $\omega_{g}$ ' is higher if ' $\omega_{0}$ ' is closer to ' $\omega_{g}$ '. Hence, the maximum lag occurs at the maximum value of ' $\omega_{0}$ '. At this condition, the value of ' $K_{1}$ ' is given by

$$
K_{1}=\tan \theta\left[\frac{\omega_{g}}{\omega_{0(\max )}}-\frac{\omega_{0(\max )}}{\omega_{g}}\right] .
$$

Equations (12) and (15) give the lower limit of ' $K_{1}$ ' and Eq. (20) gives the upper limit of ' $K_{1}$ '. In this work, value of ' $K_{1}$ ' is chosen based on these equations for ' $\omega_{0}$ ' varying in the range $10-100 \mathrm{~Hz}$. For frequencies below $10 \mathrm{~Hz}$, the PI controller gives sufficient gain $(>40 \mathrm{~dB})$. The green continuous curves in figure 11 shows the magnitude and phase plots of the open-loop transfer function between ' $I_{b a t}$ ' and ' $I_{b a t}^{*}$ after final design, while figure 12 show the close-loop gain and phase of the system. From these two figures it can be seen that the controller provides sufficient gain at dc and the frequency of measurement. Table 2 shows the chosen controller parameters for the $3 \mathrm{~kW}$ setup (figure 13) after final design.

If impedance spectroscopy is done by applying excitation containing multiple frequencies, then multiple PR controller blocks have to be used. 

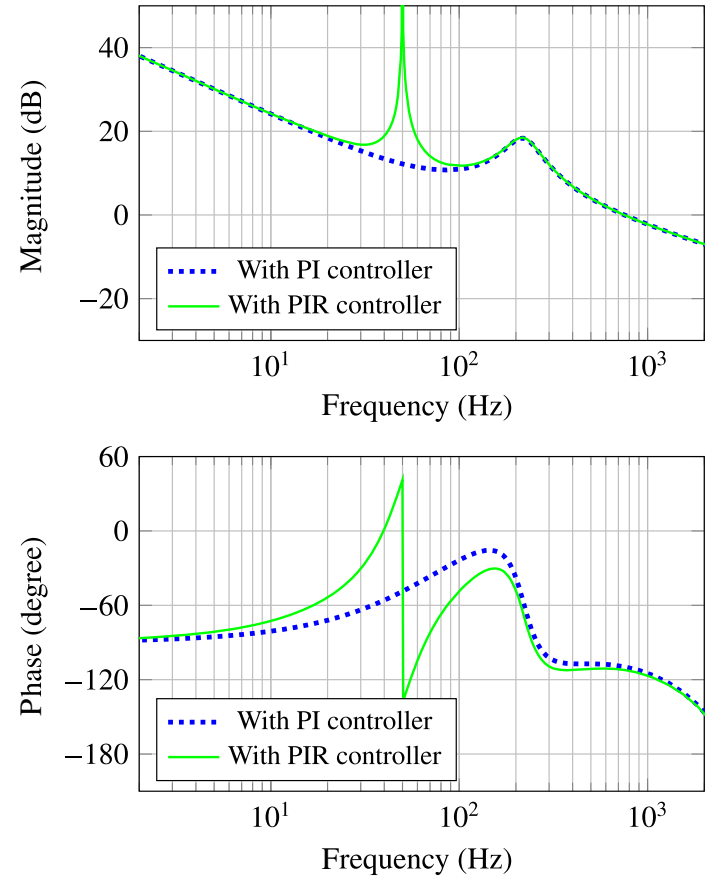

Figure 11. Magnitude and phase plots of open loop transfer function between ' $I_{b a t}$ ' and ' $I_{b a t}^{*}$ ' with PI controller (dotted blue lines) and with PIR controller (continuous green line).
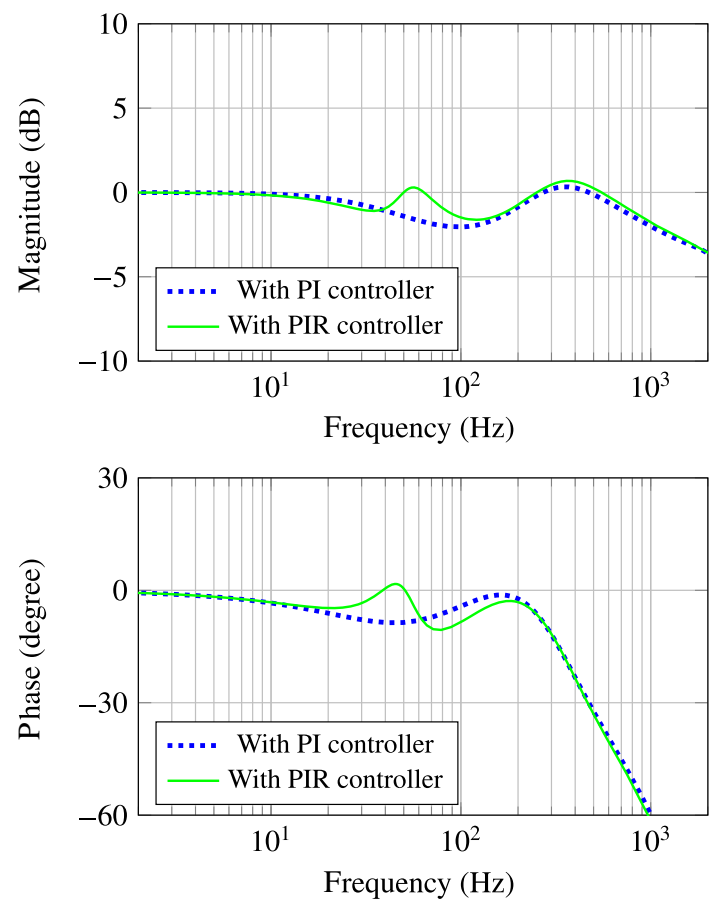

Figure 12. Magnitude and phase plots of close loop transfer function between ' $I_{b a t}$ ' and ' $I_{b a t}^{*}$ ' with PI controller (dotted blue lines) and with PIR controller (continuous green line).
Table 2. Controller parameters after final design.

\begin{tabular}{lcc}
\hline Controller & Parameter & Designed value \\
\hline Inner loop PI & $K_{p}$ & 0.073 \\
& $K_{I}$ & 43.8 \\
Outer loop PIR & $K_{p}$ & 2 \\
& $K_{I}$ & 1000 \\
& $K_{1}$ & 0.7 \\
\hline
\end{tabular}

\section{Measurement and calculation}

Measurement of voltage and current perturbation is a critical task. The difficulty comes from the fact that the current and voltage magnitudes are very small. Moreover this small voltage is added with large dc voltage. Current perturbation will also be added to a large dc quantity if measurement is done using the same power circuit that is used for charging and discharging the battery.

\subsection{Measurement circuit}

The small current and voltage signals have to be discretized and converted to digital information by analog to digital converter (ADC). Resolution of ADC depends on number of bits. To sample a very low signal, ADC needs to have high resolution using high number of bits, which leads to high cost. This additional cost can be avoided if the small signal can be amplified. If a large dc quantity is present along with the small perturbation signal then it will saturate the amplifier. A dc cancellation network is required to cancel the dc component and a low-pass filter is needed to attenuate noise. The structure of filter-amplifier circuit used in this work is shown in a block diagram form in figure 14. After amplification the signal is fed to FPGA (the digital controller) through an ADC.

The op-amps used in this circuit should have very low offset drift, otherwise the offset drift may be comparable to the voltage to be measured. In this work, low offset op-amp OP07C from TI is used, whose maximum input offset voltage is $150 \mu \mathrm{V}$ and drift of input offset voltage is $0.5 \mu \mathrm{V} /{ }^{\circ} \mathrm{C}$.

\subsection{Impedance calculation}

The real and imaginary parts of the impedance are evaluated by calculating real and imaginary parts of both current and voltage perturbations by Fourier series analysis in discrete domain at fundamental frequency implemented in FPGA. The expressions for Fourier series coefficients of a signal $x(t)$ of time period $T$ at the fundamental frequency are as follows: 


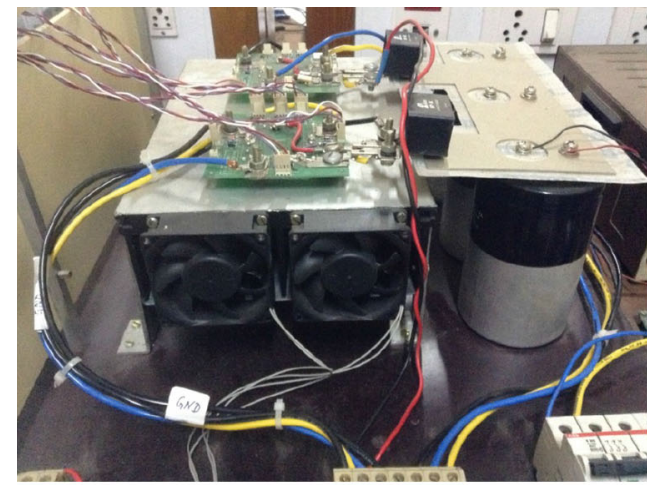

(a)

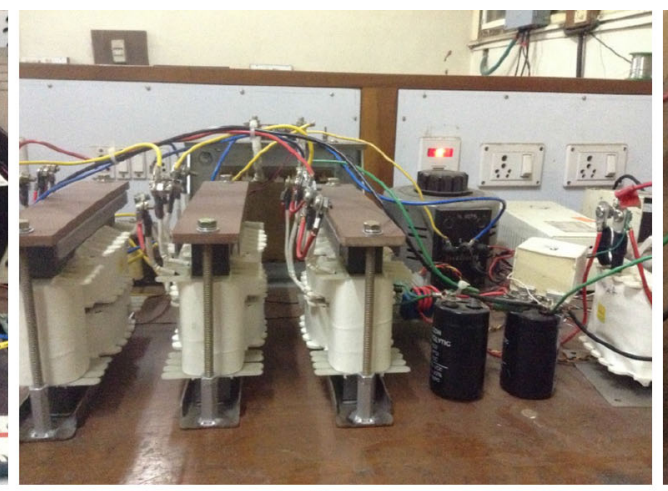

(b)

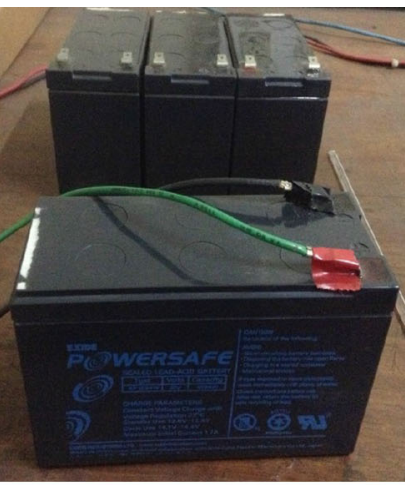

(c)

Figure 13. The hardware setup: (a) IGBT modules with heat-sink and dc bus capacitor in the bidirectional converter, (b) filter elements in the dc-dc converter and (c) battery under test.

$$
\begin{aligned}
& a_{c}=\frac{2}{T} \int_{0}^{T} x(t) \cos \left(\frac{2 \pi t}{T}\right) d t \\
& b_{c}=\frac{2}{T} \int_{0}^{T} x(t) \sin \left(\frac{2 \pi t}{T}\right) d t .
\end{aligned}
$$

Equations (21) and (22) are discretized for a sampling rate of ' $N$ ' samples per cycle. The discretized equations are given by (23) and (24), where ' $x_{k}$ ' is the ' $k$ ' th sample:

$$
\begin{aligned}
& a_{d}=\frac{2}{N} \sum_{k=0}^{N-1} x_{k} \cos \left(\frac{2 \pi k}{N}\right), \\
& b_{d}=\frac{2}{N} \sum_{k=0}^{N-1} x_{k} \sin \left(\frac{2 \pi k}{N}\right) .
\end{aligned}
$$

The amplitude and phase of signal ' $x$ ' is given by

$$
\begin{aligned}
& |x|=\sqrt{a_{d}^{2}+b_{d}^{2}}, \\
& \angle x=\tan ^{-1}\left(\frac{a_{d}}{b_{d}}\right) .
\end{aligned}
$$

Equations (23)-(26) are applied to both voltage and current perturbations to obtain their magnitude and phase. They can be used to obtain real and imaginary parts of the impedance. These calculations would be more accurate for higher value of ' $N$ '. But higher value of ' $N$ ' will need more computation resources and large memory to store the lookup table. The value of ' $N$ ' is chosen as 1024 in the hardware implementation.

\subsection{Algorithm of impedance spectroscopy}

Impedance is measured in this work by a frequency sweep from high to low frequency. The algorithm used here is shown in figure 15 . The algorithm waits before taking a measurement for the transients to die down below an acceptable level. If transients are present, calculated coefficient values of two consecutive cycles will not match. This is used to detect the steady state. If this tolerance for mismatch is very low, measurement time will increase. Hence, the choice of tolerance is a trade-off between accuracy and speed of measurement. In this work, 3\% tolerance is considered to be acceptable.

The notations and abbreviations used in flow chart are described here

IS_enable : status of the toggle switch that initiates impedance spectroscopy,

ai,bi: Fourier co-efficient of current,

av,bv: Fourier co-efficient of voltage,

read_addr: address where the frequency command is stored in a look-up table. Frequency command is read from that address and controller generates perturbation of that frequency.

write_addr: address where the co-efficients ai, bi, av and bv are stored. Separate memory blocks are used for each of the co-efficients. Hence the same address can be used for all of them.

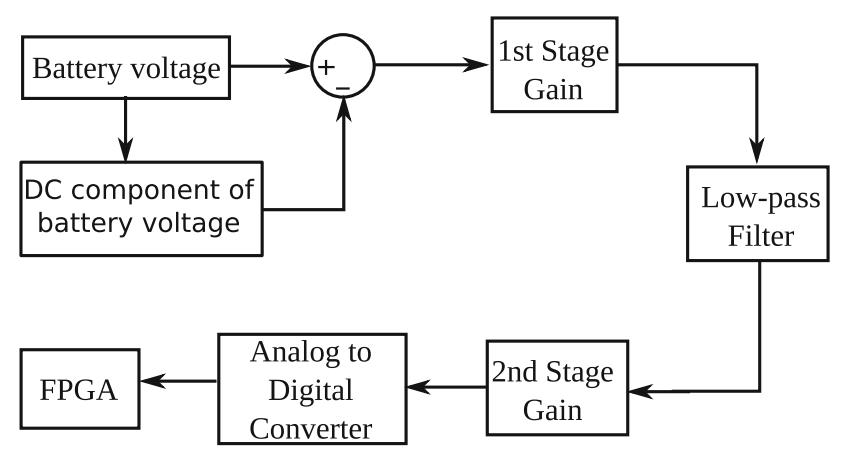

Figure 14. Block diagram of filter-amplifier circuit. 


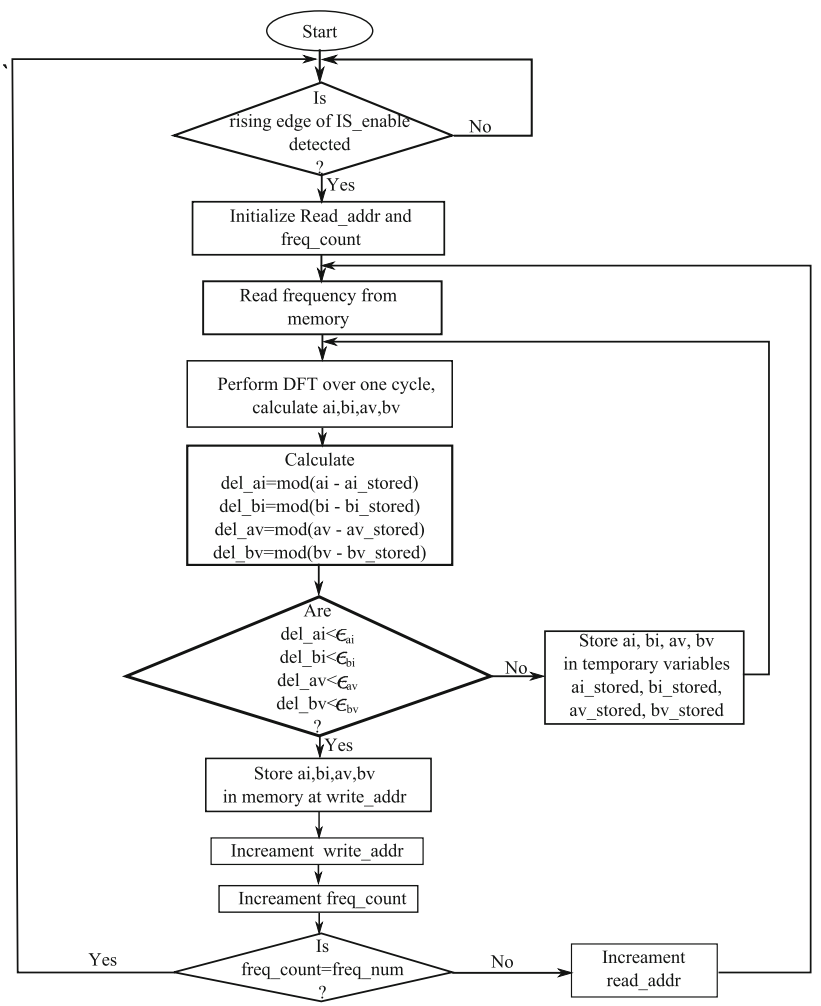

Figure 15. Flow chart of algorithm of performing impedance spectroscopy.

DFT: discrete Fourier transform. This is performed only at fundamental frequency since a single sine perturbation is used.

ai_stored, bi_stored, av_stored, bv_stored: they are the variables where ai, bi, av, bv are stored temporarily.

$\epsilon_{a i}, \epsilon_{b i}, \epsilon_{a v}, \epsilon_{b v}$ : tolerance of variation of co-efficients ai, bi, av and bv.

freq_count: number of frequencies at which impedance has been measured so far.

freq_num: total number of frequencies at which impedance has to be measured.

\subsection{Impedance of a battery bank}

If the health conditions of the batteries in a battery bank are similar and they are charged and discharged together, then their SOC and SOH values will be close to each other and overall battery bank impedance can be used for health monitoring. Such a condition exists when the batteries are new or all batteries age uniformly. If such a situation does not exist, then some battery impedance will dominate over others and overall impedance data cannot be used.

In such cases, the impedance measurement method described here can be extended to measure impedance of each battery. In order to achieve this, terminal voltage of each battery has to be measured and current of all batteries will be the same if they are connected in series. However, if

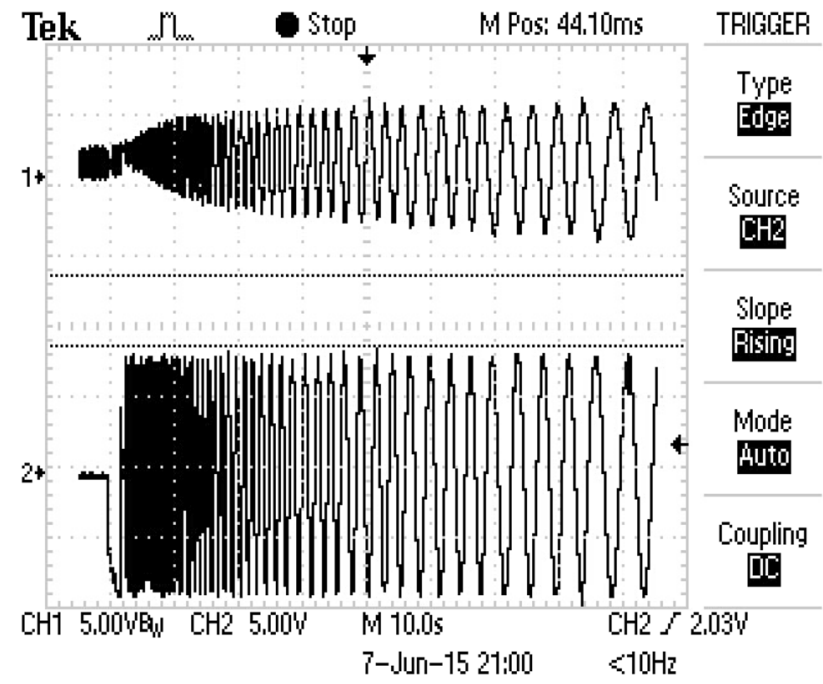

Figure 16. Waveforms during impedance spectroscopy. Channel 1: Voltage perturbation, scale $=5 \mathrm{~V} / \mathrm{div}$, sensor gain $=240$; Channel 2: current perturbation, scale $=5 \mathrm{~V} / \mathrm{div}$, sensor gain $=50$; Time scale $=10 \mathrm{~s} / \mathrm{div}$.

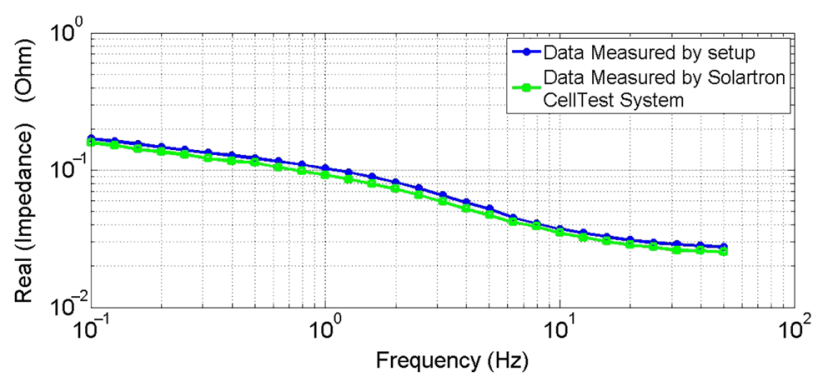

(a)

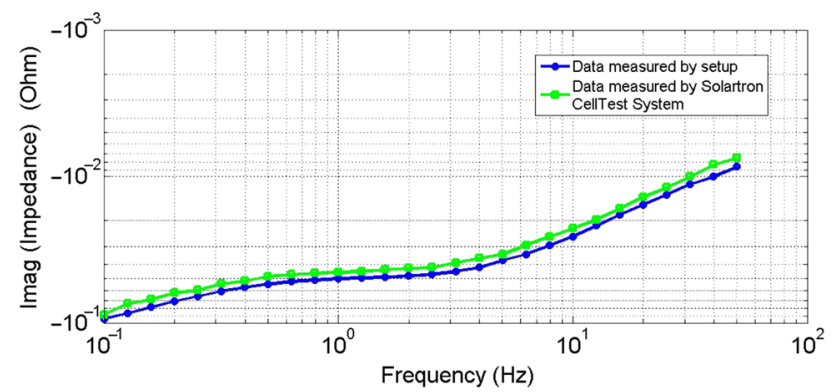

(b)

Figure 17. Comparison of impedance obtained by the converter and Solartron CellTest System [14], (a) real parts , (b) imaginary parts.

two or more such series connected battery strings are connected in parallel then current through each battery string has to be measured. Since batteries can be considered as a linear system under small perturbation, current perturbation in each string will also be sinusoidal. This sinusoidal current will cause sinusoidal perturbation in each battery voltage. Hence, individual battery impedance can be calculated from such a set-up. In this case, measuring 
each battery impedance does not affect the power circuit, but increases cost and complexity of measurement circuit.

\section{Experimental results}

Figure 16 shows the voltage and current perturbations during impedance spectroscopy. These waveforms are outputs of filter and amplifier circuits whose gains are 240 $\mathrm{V} / \mathrm{V}$ and $50 \mathrm{~V} / \mathrm{A}$. Here, the current amplitude is controlled at a fixed value.

As can be seen, the voltage amplitude increases as the frequency decreases, which shows increase of impedance with decrease of frequency. Care should be taken to choose the current amplitude so that voltage amplitude does not cross the limit of $10 \mathrm{mV} / \mathrm{cell}$ at the lowest frequency.

Figure 17 compares the impedance value measured by the set-up to that measured by the Solartron CellTest System [14] in the frequency range $0.1-50 \mathrm{~Hz}$ on a $12 \mathrm{~V}, 9 \mathrm{Ah}$ VRLA battery at $100 \%$ SOC. The results indicate a good match in the impedance trend measured using the power converter and the dedicated instruments.

\section{Conclusion}

This work implements a $3 \mathrm{~kW}$ grid-connected converter suitable for charging, discharging and characterizing a $100 \mathrm{~V}$ battery bank. The set-up can be used as a backup storage system for a grid-connected load or it can be used as a dedicated set-up for testing a battery with the provision of sending power back to grid during discharging to avoid wastage of energy. SRF-PLL has been used to estimate grid phase, which, in turn, is used in a PR-controller-based control structure to draw power from the grid at unity power factor. The provision for performing impedance spectroscopy has been implemented in the converter to eliminate the need for dedicated impedance measurement instrument. The necessity of LCL filter and double loop control structure has been discussed from the point of view of performing impedance spectroscopy. Voltage perturbation with peak less than $10 \mathrm{mV}$ has been produced using a PIR controller in the control structure of $\mathrm{dc}-\mathrm{dc}$ converter. Tuning of parameter of PIR controller has been discussed and the expressions for maximum and minimum parameter values have been derived. Measurement of small voltage and current perturbations has been done by op-amp-based filter-amplifier circuit. The real and imaginary parts of impedance are calculated by discrete Fourier transform performed in FPGA. The algorithm for taking measurement avoiding transients by a frequency sweep has been developed and implemented. The measured impedance is compared with the impedance data obtained by a commercially available impedance measuring equipment. This feature of impedance measurement allows impedance-spectroscopy-based health monitoring of the battery with the power converter eliminating any need for expensive dedicated commercial instruments.

\section{References}

[1] Barsoukov E and Macdonald J R 2005 Impedance spectroscopy: theory, experiment and application, 2nd edn. Wiley-Interscience, Hoboken

[2] Kai S and Qifang S 2011 Overview of the types of battery models. In: Proceedings of the 30th Chinese Control Conference

[3] Rezvanizaniani S M, Liu Z, Chen Y and Lee J 2014 Review and recent advances in battery health monitoring and prognostics technologies for electric vehicle (EV) safety and mobility. J. Power Sources 256: 110-124

[4] Barr A, Deguilhem B, Grolleau S, Grard M, Suard F and Riu D 2013 A review on lithium-ion battery aging mechanisms and estimations for automotive applications. J. Power Sources 241: 680-689

[5] Zhang J and Lee J 2011 A review on prognostics and health monitoring of Li-ion battery. J. Power Sources 196: $6007-6014$

[6] Huet F 1998 A review of impedance measurements for determination of the state-of-charge or state-of-health of secondary batteries. J. Power Sources 70: 59-69

[7] Shalini Rodrigues, Munichandraiah N and Shukla A K 2000 A review of state-of-charge indication of batteries by means of a.c. impedance measurements. J. Power Sources 87: 12-20.

[8] Christophersen J P, Glenn D F, Motloch C G, Wright R B, Ho C D and Battaglia V S 2002 Electrochemical impedance spectroscopy testing on the Advanced Technology Development Program lithium-ion cells. Vehicular Technology Conference. In: Proceedings 56th IEEE VTC 2002-Fall, pp. 1851-1855

[9] Blanke H, Bohlen O, Buller S, Doncker R W D, Fricke B, Hammouche A, Linzen D, Thele M and Sauer D 2005 Impedance measurements on leadacid batteries for state-ofcharge, state-of-health and cranking capability prognosis in electric and hybrid electric vehicles. J. Power Sources 144: 418-425

[10] Huang W and Qahouq J A 2014 An online battery impedance measurement method using DC-DC power converter control. IEEE Trans. Ind. Electron. 61(11): 5987-5995

[11] Kulkarni A and John V 2013 Analysis of bandwidth-unitvector-distortion tradeoff in PLL during abnormal grid conditions. IEEE Trans. Ind. Electron. 16: 5820-5829

[12] Parikshith B C 2009 Integrated approach to filter design for grid connected power converters, Masters Thesis, Indian Institute of Science, Bangalore

[13] Barsoukov E, Kim J H, Hwang K S, Yoon C O and Lee H 2000 Parametric analysis using impedance spectroscopy: relationship between material properties and battery performance. J. New Mater. Electrochem. Syst. 3: 303-310

[14] Solartron Analytical Solartron CellTest System. http://www. solartronanalytical.com/download/Model-1470E-Brochure. pdf 
[15] Tröltzsch U, Kanoun O and Tränkler H R 2006 Characterizing aging effects of lithium ion batteries by impedance spectroscopy. Electrochim. Acta 51(89): 1664-1672

[16] Ghoshal A and John V 2015 A controller design method for 3 phase 4 wire grid connected VSI with LCL filter. Sadhana 40(5): 1481-1499

[17] Zmood D N and Holmes D G 2003 Stationary frame current regulation of PWM inverters with zero steady-state error. IEEE Trans. Power Electron. 18(3): 814-822
[18] Exide Industrial Power Safe EP Series Application Note. www.exideindustrialbatteries.com/file/EP.PDF

[19] Ghoshal A and John V 2011 Design of current controlled three phase grid connected VSI for operation under non-ideal grid condition. In: Proceeding of the Centenary Conference, Indian Institute of Science, Bangalore

[20] Ghosal A 2014 Operation of three phase four wire grid connected VSI under non-ideal conditions. PhD Thesis, Indian Institute of Science, Bangalore 